\title{
APC-resistance Is a Risk Factor for Postoperative Thromboembolism in Bective Replacement of the Hip or Knee - A Prospective Study
}

\author{
Toma s L. Lindahl' ${ }^{1}$, Tom H. Lunda hl2, Lenna rt Nilsson ${ }^{3}$, C hrister A. Andersson ${ }^{4}$ \\ From the Departments of ${ }^{1} \mathrm{C}$ linic al Chemistry, University Hospital, Linköping, \\ 2Vä stervik Hospita I, Västervik, ${ }^{3}$ Northem Älvsborg Hospital, Tro llhättan, and ${ }^{4}$ Department of Orthopaedic Surgery, \\ University Hosp ital, Linköping, Swed en
}

\section{Summary}

Postoperative venous thromboembolic complications are commonly seen after total replacement of the hip or knee. Recently, an inherited defect with resistance to the anticoagulant activity of activated protein C (APC-resistance) has been detected. APC-resistance seems to be a common risk factor, especially in Sweden, and it increases the propensity for venous thrombosis. This study assesses the prevalence of APCresistance in a general population and its clinical significance for patients undergoing surgery associated with a high risk of thromboembolic complications. In a prospective cohort study, we analysed for APCresistance in 645 consecutive patients before elective replacement of the hip or knee at 3 hospitals in southern Sweden. Thromboprophylaxis with LMWH-heparin was given to all patients throughout the hospitalisation period. We recorded events of clinical thromboembolism for 3 months postoperatively. Venography, ultrasonography or pulmonary scintigraphy was requested by the clinicians according to the existing routines, i.e. only patients with symptoms of thromboembolism were examined. A thromboembolic complication was registered in $20(3.1 \%)$ patients. Fifty per cent of the venous thrombi had a proximal location. Only $0.3 \%$ of the patients had verified pulmonary embolism. APC-resistance was found in $14.1 \%$ of the patients, of whom $9.9 \%$ had experienced postoperative thromboembolism compared with $2.0 \%$ of the patients without APC-resistance ( $\mathrm{p}<0.0007)$. We conclude that APCresistance is a frequent risk factor for symptomatic postoperative deep venous thrombosis with an estimated relative risk of 5.0 (95\% confidence interval: from 1.9 to 12.9) in elective replacement of the hip or knee.

\section{Introduction}

Replacement of the hip or knee carries a high risk of postoperative deep venous thrombosis (DVT) and the incidence of thromboembolic complications has been reported to be within the range of 50 to $60 \%$ without the use of prophylactic anticoagulation therapy (1). This high complication rate is considerably reduced with subcutaneous injections of low molecular-weight heparin (LMWH) administered during the routine hospitalisation period of one week (2). However, in recent reports a protracted risk of thromboembolism for at least one month postoperatively after total hip replacement has been described. By prolongation of the prophylaxis using LMWH a significant reduction of venography screened DVT was demonstrated in recent studies (3-6).

Correspondence to: Tomas L. Lindahl, MD PhD, Department of Clinical Chemistry, University Hospital, S-58185 Linköping, Sweden - Tel.: +46 132232 27; FAX: +46 1322 32 40; E-mail: tomas.lindahl@lio.se
Furthermore, the health care costs for long-term complications of DVT are considerable (7). The protein C-anticoagulant system is an important mechanism for the physiological control of the coagulation system. Resistance to the anticoagulant activity of the activated protein $\mathrm{C}$ (APC-resistance) is an inherited defect of this system (8-9). In approximately $95 \%$ of the cases the cause can be attributed to a single point mutation in coagulation factor $\mathrm{V}\left(\mathrm{FV}: \mathrm{Q}^{506}\right)(10)$. This mutation is relatively common with carrier rates in Caucasians ranging from 5 to 15\% (11-12). The highest incidence of APC-resistance has been found in Sweden (11-13). In several studies, the relative risk of thrombosis in individuals with APC-resistance has been estimated to be in the order of 2 to 10 (14-16). Since most studies are based on cases referred to centres for investigation, these frequencies may be an overestimation due to a preferential referral of young patients with a family history of thromboembolic disease. A recent study indicated that APC-resistance is a potential risk factor for thrombosis after elective hip arthroplasty (13). The patients were subjected to venography 5 weeks postoperatively. The objective of the present study was to assess APC-resistance in a population scheduled for replacement of the hip or knee, and to investigate if APC-resistant patients carried an increased risk of symptomatic postoperative venous thrombosis.

\section{Material and Methods}

Patient cohort. The study included 645 consecutive patients who had elective replacement of the hip or knee at hospitals in Ljungby, Västervik and Växjö, Sweden during a period of 20 months, between September 1994 and May 1996. Blood samples were collected from the patients for subsequent analysis of APC-resistance, excluding those on therapeutic oral anticoagulants. Informed consent was obtained from all patients and the study protocols were approved by the ethics committee.

APC-resistance analysis. The separated plasma was stored at $-80^{\circ} \mathrm{C}$ for subsequent measurement of APC-resistance. Analysis was performed after 1:4 predilution of the patient plasma in factor V-deficient plasma according to the manufacturer's instructions (Chromogenix AB, Mölndal, Sweden). In a recent evaluation, the method showed a sensitivity of $100 \%$ and specificity of $98.8 \%$ for the FV R506Q-mutation (17). We have the same favourable experience from thrombophilia work-up samples, which were also analysed for the factor $\mathrm{V}$ mutation during the same time period.

Study design and surgery. Study protocols were completed prior to surgery, at the onset of symptoms of thrombosis, on discharge from the hospital (usually at one week postoperatively) and at a follow-up examination at approximately 3 months postoperatively. Thromboprophylaxis by subcutaneous injections of LMWH in the abdominal wall was given once daily to all patients throughout the hospitalisation period with the first dose administered the evening before surgery. At one centre (Västervik), $5000 \mathrm{IU}$ of anti-Xa dalteparinsodium (Fragmin, Pharmacia-Upjohn, Uppsala, Sweden) was given and at the other two hospitals (Växjö, Ljungby), $4000 \mathrm{IU}$ of anti-Xa enoxaparin (Clexane, Rhône-Poulenc-Rorer, Collegeville, PA). 
Table 1 Incidence of postoperative thromboembolic events confirmed by objective methods, APC-resistance and self-reported previous thrombosis. The estimated risk-ratio is expressed with a $95 \%$ confidence interval (C.I.). APC-resistance was measured with samples prediluted in factor $\mathrm{V}$-deficient plasma

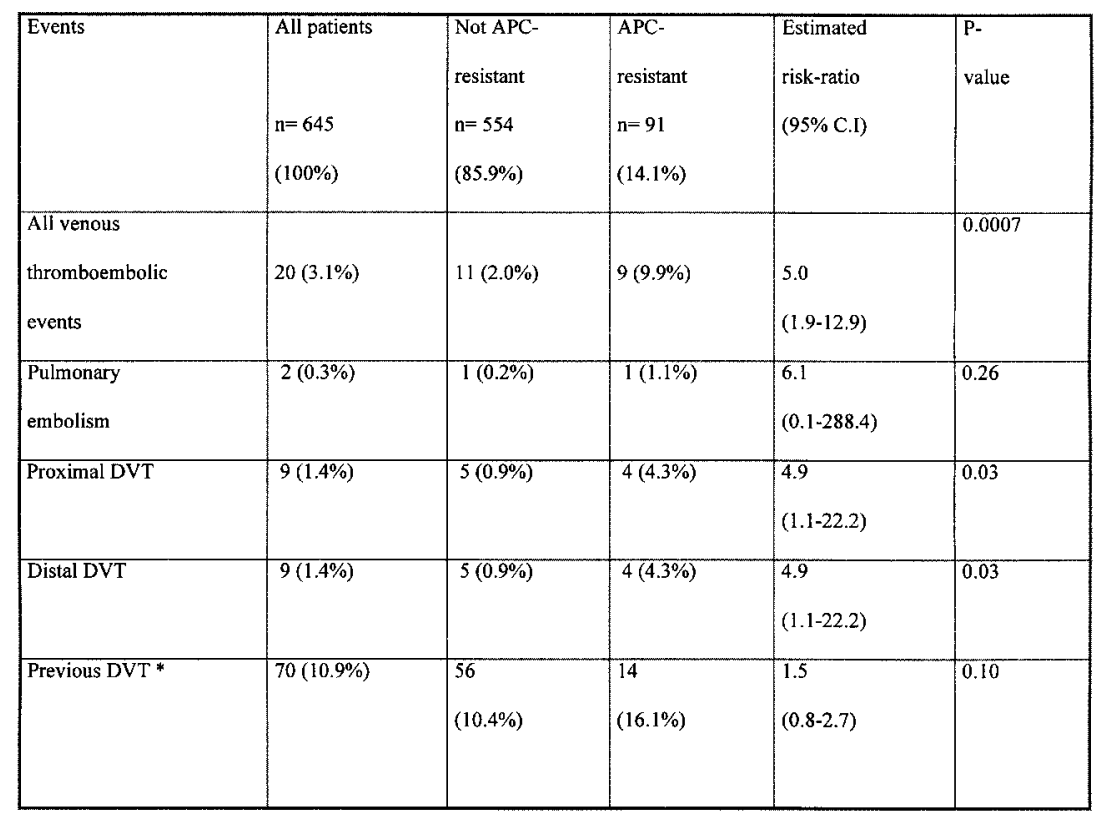

* 629 patients answered this question
The replacements were performed under general or spinal anaesthesia using a conventional surgical technique and cemented components. For the hip replacements, a posterior approach was used with the patients resting on the contralateral side whereas the knee replacement, the patients were supine, a tourniquet was used, and either an unicondylar or bicondylar prosthesis was inserted. Rehabilitation, in the form of passive motion exercises, was initiated one day postoperatively and active motion and weight bearing were allowed when the patients had sufficient muscular control of the leg.

Diagnosis and follow-up. We recorded events of clinical thromboembolism for 3 months postoperatively. Venography, ultrasonography or pulmonary scintigraphy was requested by the clinicians according to the existing routines, i.e. only patients with symptoms of thromboembolism were examined. All of the thrombi were confirmed by venography. An intraluminal filling defect of the deep venous system was considered to be a direct sign of thrombosis. Partial or non-filling of the deep veins not due to technical reasons was interpreted as an indirect sign of thrombosis unless signs of a severe postthrombotic state rendered the interpretation impossible. Thrombosis in, or proximal to, the popliteal vein was regarded as proximal and thrombosis distal to the popliteal vein, as distal. Moreover, all venographies, ultrasonographies and pulmonary scintigraphies performed at the participating centres between September 1994 and August 1996 were checked to ensure that all complications had been included. Based on hospital records, a central consensus on the causes of death was also established.

Statistics. The results of our investigation are expressed in terms of risk ratios. Confidence intervals were calculated using the standard procedure, which involved a logarithmic transformation of the observed risk ratio and an approximation of the probability distribution of the transformed variable by a normal distribution (18). Continuity corrections were applied. The significance of the differences between the APC-resistant and the non-resistant groups were determined using the Fisher exact test in its one-sided version.

\section{Results}

In our study the number of patients included with a total hip or knee replacement was 424 and 221, respectively. There were 271 (42.0\%) men and $374(58.0 \%)$ women with a mean age of $70.5 \pm 9.9$ years. There was no difference in age between the patients with a hip or knee replacement, nor between those with or without APC-resistance. There were no losses during the follow-up. Of the 645 patients evaluated,
$20(3.1 \%)$ patients had a thromboembolic complication confirmed by objective methods (Table 1), 18 (2.8\%) of whom had deep vein thrombosis, and $2(0.3 \%)$ had pulmonary embolism. The deep vein thrombi were predominantly $(89 \%)$ found in the operated leg. A proximal location of the thrombosis was as common as a distal. Sixteen (3.8\%) of the patients with postoperative thromboembolism had undergone a hip replacement, and 4 (1.8\%; nonsignificant), a knee replacement. Of 374 women, $13(3.5 \%)$ suffered venous thrombosis compared with 7 (2.6\%) out of 271 men (nonsignificant).

The prevalence of APC-resistance among all of the patients was $14.1 \%$ (Table 1) and $9.9 \%$ of these cases had postoperative thromboembolism, compared with $2.0 \%$ of the patients without APC-resistance ( $p$ <0.0007). Thus, the estimated relative risk was 5.0 (95\% confidence interval: 1.9 to 12.9) for patients with APC-resistance and the corresponding estimated relative risk for venous thrombosis of the leg was $4.9(p=0.03)$. Six patients of the non-APC-resistant patients had a proximal DVT or pulmonary embolism $(1.1 \%)$ as well as 5 of the APCresistant $(5.5 \%)$, i.e. the relative risk for pulmonary embolism or proximal thrombosis is 5.1 (significant) and that for pulmonary embolism, 6.1 (nonsignificant).

The thromboembolic complications were diagnosed between 6 and 51 days postoperatively. Sixteen $(80 \%)$ of the 20 patients were diagnosed within 35 days of the operation and four, one with APC-resistance and 3 without, had a thrombosis diagnosed during the first postoperative week, i.e. when they received prophylactic treatment. Another 25 patients, 3 (12\%) of them with APC-resistance, were examined due to clinically suspected thromboembolic disease, but the diagnosis was ruled out in all patients by venography. Among the 629 patients who had answered the question as to whether they had any previous thromboembolic problems the prevalence of APC-resistance was $16.1 \%$ compared with $10.4 \%$ for the patients without APC-resistance. This gives an estimated risk-ratio of 1.5 (nonsignificant) (Table 1). Of 19 patients with a confirmed postoperative thrombosis, only $3(15.8 \%)$ reported previous thromboembolic events. Thus, 67 (10.7\%) patients with a history of thromboembolism did not develop any thromboembolic complications, which gives an estimated risk-ratio of 1.5. During the follow-up period, four patients, one of whom was APC-resistant, died 
due to myocardial infarction (1 case) and nondetermined causes ( 3 cases). Two patients experienced cerebrovascular lesions prior to the follow-up visit and died shortly thereafter.

\section{Disc ussion}

This prospective study, with the endpoints being death or clinical symptoms of thromboembolism confirmed by objective methods, demonstrated a five-fold increased risk of postoperative DVT for patients with APC-resistance. APC-resistance, which was determined using a functional method with high sensitivity and specificity for the factor $\mathrm{V} \mathrm{Q}^{506}$ mutation (17), was found in one out of every seven cases in our population of consecutive patients. Although the APC-resistant patients had a higher risk of DVT, they did not show any significant increase of the risk of stroke, myocardial infarction or death of any cause during the observation period of 3 months.

In a recent Swedish study (13) on elective hip replacement and prolonged prophylaxis with LMWH in which the patients were screened by venography 21 days postoperatively, an increased risk of thrombosis for $\mathrm{FV} \mathrm{Q}^{506}$ carriers was found only for the subgroup given standard prophylaxis, i.e. during hospitalisation. There was no information provided on the incidence of symptomatic thrombosis. In contrast to our study, there was a higher prevalence of APC-resistance among women. We believe, however, that our larger patient material is likely to give a better estimate of the true incidence. However, a gender influence on asymptomatic thrombosis cannot be ruled out by our study. In a retrospective study by Ryan et al.(19), which included 825 patients, a prevalence of the FV $\mathrm{Q}^{506}$ mutation was observed in $3.9 \%$ of the patients with various kinds of prophylaxis after elective hip or knee surgery with screening by venography 5-9 days postoperatively, they did not find an increased risk of postoperative thrombosis among $\mathrm{FVQ}^{506}$ carriers. In comparison with our study the main differences compared are the endpoints: venography instead of symptomatic thrombosis confirmed by objective methods and the observation time of one week instead of 3 months.

The incidence of clinically overt thromboembolism in our study was low in comparison with recent studies in which all patients were screened for thrombosis with objective methods (3-6). Nevertheless, we believe that we have documented most of the severe thromboembolic complications, since a meticulous control of all registered examinations for thromboembolic diseases was carried out at the participating centres. It is well known that asymptomatic thromboembolism is far more common, but its relevance as a significant clinical complication can be questioned since screening of all patients is not routinely performed anywhere. Moreover, evidence supporting prophylactic treatment of asymptomatic thrombosis is, to the best of our knowledge, lacking.

Despite a high mean age and a considerable relative risk for thromboembolism, it was surprising to find that there was a similar selfreported frequency of previous thromboembolism in APC-resistant patients and nonresistant patients. A history of previous thromboembolic disease alone had a low predictive value for the risk of postoperative thrombosis. One might speculate that in a nonselected population, APC-resistance carries an increased risk of thrombosis only if there are other coexisting risk factors, genetic or circumstantial. The predominance of venous thrombosis in the operated leg indicates the importance of local factors. In the present study, the complication rate after a hip arthroplasty was not higher than after a knee arthroplasty. In the present investigation, the mortality rate was $0.9 \%$ during an observation period of 4 months. This is a comparatively high incidence and the risk for a fatal outcome after major arthroplastic surgery of the hip or knee in an elderly population should not be overlooked. It is possible that the incidence of pulmonary embolism has been underestimated.

Recently, some authors have recorded a prolonged thrombogenic period after a total hip replacement. Bergqvist et al. and Dahl et al. evaluated prophylaxis with LMWH for 4 and 5 weeks, respectively, instead of just during the period of hospitalisation (3-4). In our study, $80 \%$ of the thromboembolic complications were clinically overt and diagnosed within 5 weeks of the operation. From clinical experience, it is well known that prophylaxis with LMWH does not eliminate the risk of thromboembolic complications, and $20 \%$ of the thrombotic events in our study were diagnosed during the first postoperative week when the patients were still receiving prophylactic treatment.

In this study, APC-resistance was found to be a common risk factor with a high clinical significance. In order to further evaluate its clinical significance, it seems reasonable to perform a controlled study with LMWH injections for 5 weeks in patients und ergoing major arthroplastic surgery of the leg, comparing APC-resistant with nonresistant patients. A more individualised prophylactic regimen tends to optimise the outcome and is also likely to be beneficial from an economic point of view.

\section{Acknowledgements}

We would like to express our gratitude to Mrs Gull-Britt Andersson, Ms Inger Fagerberg and Ms Catharina Sjöberg for their technical assistance. The contributions made by Martin Fagerlund, MD, Växjö Hospital, Dan Linvik, MD, Ljungby Hospital and Örjan Öst, MD, Västervik Hospital and their respective staff of the departments of Orthopaedic Surgery, as well as the staff of the clinical chemistry laboratories at the hospitals in Växjö, Ljungby and Västervik, are also gratefully acknowledged. We wish to thank Professor Erik Leander for statistical advice and Mrs Doris Schulman for linguistic revision. Chromogenix AB, Mölndal, Sweden, provided reagents for the analysis of APC-resistance.

\section{References}

1. Turpie AGG, Levine MN, Hirsch J, et al. A randomized controlled trial of a low-molecular-weight heparin (enoxaparin) to prevent deep vein thrombosis in patients undergoing elective hip surgery. N Engl J Med 1986; 315 : 925-9.

2. Bergqvist D. Review of clinical trials of low molecular weight heparins. Clinical review. Eur J Surg 1992; 158: 67-8.

3 Bergqvist B, Benoni G, Björgell O, et al. Low-molecular-weight heparin (enoxaparin) as prophylaxis against venous thromboembolism after total hip replacement. N Engl J Med 1996; 335: 696-700.

4. Dahl O E, Andreassen G, Aspelin T, et al. Prolonged thromboprophylaxis following hip replacement surgery - results of a double-blind, prospective, randomised, placebo-controlled study with dalteparin $\left(\right.$ Fragmin $\left.^{\circledR}\right)$. Thromb Haemost 1997; 77: 26-31.

5. Planes A, Vochelle N, Darmon JY, Fagola M, Huet Y. Risk of deep venous thrombosis after hospital discharge in patients undergoing total hip replacement. Double-blind randomised comparison of enoxaparin versus placebo. Lancet 1996; 348: 224-8.

6. Lassen MR, Borris LC, on behalf of the Danish Prolonged Thromboprophylaxis Study Group. Prolonged thromboprophylaxis with low molecular weight heparin (Fragmin) after total hip arthroplasty- a placebo-controlled study. Thromb Haemost 1995; 73: 1104.

7. Bergqvist D, Jendteg S, Johansen L, Persson U, Ödegaard K. Cost of long-term complications of the deep venous thrombosis of the lower extremities: An analysis of a defined patient population in Sweden. Ann Inter Med 1997; 126: 454-7.

8. Dahlbäck B, Carlsson M, Svensson PJ. Familial thrombophilia due to a previously unrecognised mechanism characterized by a poor anticoagulant 
response to activated protein $\mathrm{C}$ : prediction of a cofactor to activated protein C. Proc Nat Acad Sci 1993; 90: 1004-8.

9. Svensson P J, Dahlbäck B. Resistance to activated protein C as a basis for venous thrombosis. N Engl J Med 1994; 330: 517-22.

10. Bertina RM, Koeleman BPC, Koster T, Rosendaal FR, Dirven RJ, de Ronde H, van der Velden PA, Reitsma PH. Mutation in blood coagulation factor $\mathrm{V}$ associated with resistance to activated protein $\mathrm{C}$. Nature 1994; 369: 64-7.

11. Rees DC, Cox M, Clegg JB. World distribution of factor V Leiden. Lancet 1995; 346: 1133-4.

12. Holm J, Zöller B, Berntorp E, Erhardt L, Dahlbäck B. Prevalence of factor $\mathrm{V}$ gene mutation amongst myocardial infarction patients and healthy controls is higher in Sweden than in other countries. J Int Med 1996; 239 : 221-6.

13. Svensson PJ, Benoni G, Fredin H, Björgell O, Nilsson P, Hedlund U, Nylander G, Bergqvist D, Dahlbäck B. Female gender and resistance to activated protein $\mathrm{C}\left(\mathrm{FV}: \mathrm{Q}^{506}\right)$ as potential risk factors for thrombosis after elective hip arthroplasty. Thromb Haemost 1997, 78: 993-6.

14. Bloemenkamp KWM, Rosendaal FR, Helmerhorst FM, Büller HR, Vandenbroucke JP. Enhancement by factor V Leiden mutation of risk of deep-vein thrombosis associated with oral contraceptives containing a third-generation progestagen. Lancet 1995; 346: 1593-6.
15. Ridker PM, Hennekens $\mathrm{CH}$, Lindpainter $\mathrm{K}$ et al. Mutation in the gene coding for coagulation factor $\mathrm{V}$ and the risk of myocardial infarction, stroke and venous thrombosis in apparently healthy men. New Engl J Med 1995; 332: 912-7.

16. Dahlbäck B, Hillarp A, Rosen S, Zöller B. Resistance to activated protein C, the FV: $\mathrm{Q}^{506}$ allele, and venous thrombosis. Am Hematol 1996; 72: $166-76$.

17. Svensson P J, Zöller B, Dahlbäck B. Evaluation of original and modified APC-resistance test in unselected outpatients with clinically suspected thrombosis and in healthy controls. Thromb Haemost 1997; 77: 332-5.

18. In: Altman DG, ed. Practical statistics for medical research, $1^{\text {st }}$ edn. London, UK: Chapman \& Hall, 1996: 229-72.

19. Ryan DH, Mark AC, Ginsberg JS, Francis CW. Relation of Factor V Leiden genotype to risk for acute deep venous thrombosis after joint replacement surgery. Ann Intern Med 1998; 128: 270-6.

Received April 1, 1998 Accepted after revision October 8, 1998

DISCLAIMER Published materials reflect the authors' opinions only, not those of the editor of the journal or the publisher. Neither the editor nor the publisher assume any warranty for the correctness of the opinions or representations made by the authors.

No conclusion as to whether it is a question of registered or non-registered trade marks may be drawn from the mentioning of trade names or marks in this journal. 